\title{
Adjuvant treatments for biliary atresia
}

\author{
Jessica Burns, Mark Davenport \\ Department of Paediatric Surgery, King's College Hospital, London, UK \\ Contributions: (I) Conception and design: M Davenport; (II) Administrative support: None; (III) Provision of study materials or patients: None; (IV) \\ Collection and assembly of data: All authors; (V) Data analysis and interpretation: All authors; (VI) Manuscript writing: All authors; (VII) Final \\ approval of manuscript: All authors. \\ Correspondence to: Prof. Mark Davenport, ChM, FRCS (Paeds). Department of Paediatric Surgery, King's College Hospital, London SE5 9RS, UK. \\ Email: markdav2@ntlworld.com.
}

\begin{abstract}
The treatment of biliary atresia (BA) is predominantly surgical with firstly an attempt at restoration of bile flow from the native liver by wide excision of the obstructed, obliterated extrahepatic biliary tree to the level of the porta hepatis and a portoenterostomy using a long Roux loop-Kasai portoenterostomy (KPE). Liver transplantation is reserved for those that fail this and for those where surgery is considered futile for reasons of age or stage of disease. As the aetiology of BA remains ill-defined, so adjuvant treatment has been largely based on pragmatism, trial and error. Systematic analysis of the few randomized placebo-controlled trial data and less well-controlled cohort studies have suggested benefit from post-operative high-dose steroids and ursodeoxycholic acid (UDCA) while the benefit of long-term prophylactic antibiotics, bile acid sequestrants (e.g., colestyramine) or probiotics remains unproven. Newer modalities such as antiviral therapy (AVT), immunoglobulin, FXR agonists (e.g., obeticholic acid), ileal bile acid transporter (IBAT) antagonists (e.g., maralixibat) remain unproven. This article reviews the current evidence for the efficacy of adjuvant medical therapy in BA.
\end{abstract}

Keywords: Biliary atresia (BA); adjuvant therapy; corticosteroids; ursodeoxycholic acid (UDCA); cholangitis

Submitted Aug 08, 2016. Accepted for publication Apr 14, 2020.

doi: $10.21037 /$ tp.2016.10.08

View this article at: http://dx.doi.org/10.21037/tp.2016.10.08

\section{Introduction}

Biliary atresia (BA) is a rare disease of the newborn that causes persistent conjugated jaundice, together with pale stools and dark urine; and, if left untreated can cause fibrosis, cirrhosis and ultimately end-stage liver disease within the first year of life (1).

Its overall incidence varies across the world from 1 in $5,000-10,000$ in Taiwan and Japan (2,3) to 1 in 15,00020,000 in Europe and North America (4-6). The reason for the disparity is not at all known but may be due to differences in genetic susceptibility or differing exposure to viral pathogens. Interestingly, other conditions such as congenital choledochal malformations also seem show to the same trend, though there is no real suggestion that they are aetiologically related.

Pathologically it is an obstructive cholangiopathy affecting both the intra- and extra-hepatic bile ducts to varying degrees. Sometimes there is a marked inflammatory component, while in others there is simply absence of parts of the extrahepatic bile duct system. Fibrosis is agerelated and not present at the time of birth but becomes increasingly obvious beyond 3-4 months of life. This is invariably accompanied by portal hypertension and splenomegaly. The most proximal level of obstruction is used to characterize the type of BA with $>90 \%$ having an obstruction at the level of the porta hepatis (type 3). Less commonly the obstruction is at the level of the common hepatic duct (type 2) and common bile duct (type 1). It is also possible to have cystic change within an otherwise obliterated biliary tree (so-called cystic BA) which may even contain bile, though it is unrelated to an actual congenital choledochal malformation.

The aetiology of BA is not fully understood however it 


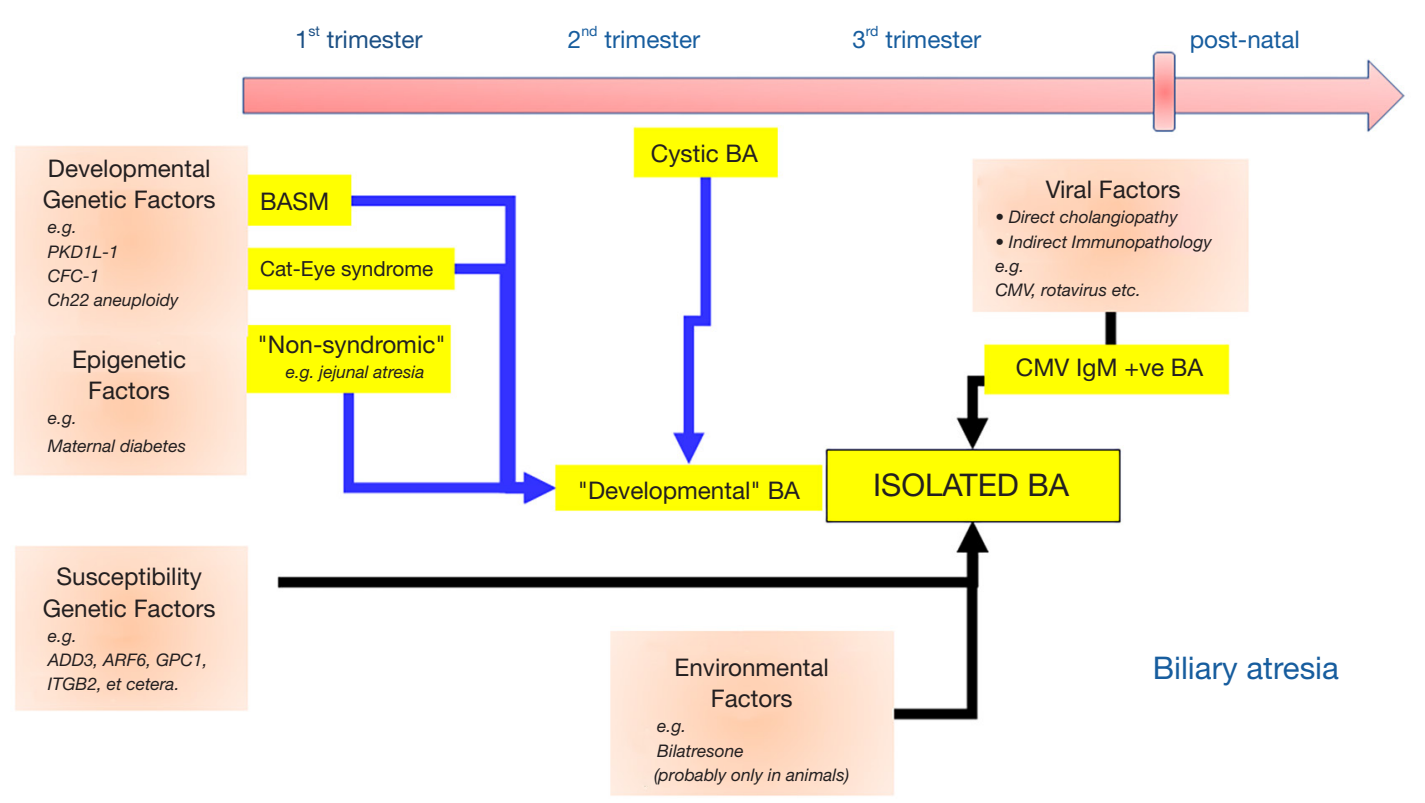

Figure 1 Potential mechanisms and timelines for biliary atresia.

is very unlikely that there is single underlying cause for all cases; rather a number of different mechanisms and factors are thought to lead to a final common pathway that is the recognizable as the BA phenotype, a phenomenon known as aetiological heterogeneity (7) (Figure 1). We have described from experience at King's College Hospital a number of distinct variants (Table 1) including a syndromic form biliary atresia splenic malformation (BASM) $(8,9)$ cystic BA $(10)$ and more recently CMV IgM +ve associated BA $(11,12)$. The remaining cases, indeed the majority, do not have any other defining characteristics and are referred to as isolated BA. One can infer that certainly for those cases with BASM (and those with other non-syndromic anomalies) and cystic BA that there is developmental derangement within the first and second trimester respectively. On the other hand, in those with perinatal CMV exposure, and hence IgM (infant-origin) antibodies, the current suggestion is that the hepatotropic virus triggers an immune-mediated injury in a normally developed bile duct, though actual evidence in the human condition for this is very limited. This concept has replaced the previous dichotomy between "embryonic" and "perinatal" BA, which is simply naive.

Over the past few years there has been a major breakthrough in understanding of timing in BA. This followed from hard clinical data from Houston, Texas where they looked back at blood taken on days 1 and 2 of life from their clinical series of infants later diagnosed as having BA (13).
They found that although the measured total bilirubin was no different from controls, the direct (conjugated) bilirubin levels were completely discriminatory. The clear implication was that at the time of birth their bile ducts were already obstructed.

The clinical presentation of BA is typically with progressive conjugated jaundice, pale acholic stools and dark urine. Other early presenting features may include a vitamin $\mathrm{K}$ dependent coagulopathy; with later ones being, ascites, portal hypertension and splenomegaly. Pre-operative diagnosis is possible but there are many protocol variations between different centres. Standard tests include liver biochemistry, ultrasonography and exclusion of medical causes such as $\alpha$-1-antitrypsin deficiency, cystic fibrosis, Alagille's syndrome, etc. Other more contentious tests include dynamic radioisotope biliary imaging, duodenal bile aspiration, ERCP and percutaneous liver biopsy (14). Our preference at King's is still for percutaneous liver biopsy and the histological features of note are portal tract oedema, bile duct plugging and proliferation together with a small cell infiltrate and occasionally giant cell formation.

Early diagnosis and prompt Kasai portoenterostomy (KPE) are essential for the best outcomes with the aim of surgery being to restore bile flow, reduce clinical jaundice and (hopefully) reverse liver fibrosis, thus salvaging the native liver. The operation removes the entire obliterated extrahepatic biliary remnant leaving the denuded (still often apparently solid) portal plate which is then anastomosed 
Table 1 The variants of biliary atresia: aetiological heterogeneity

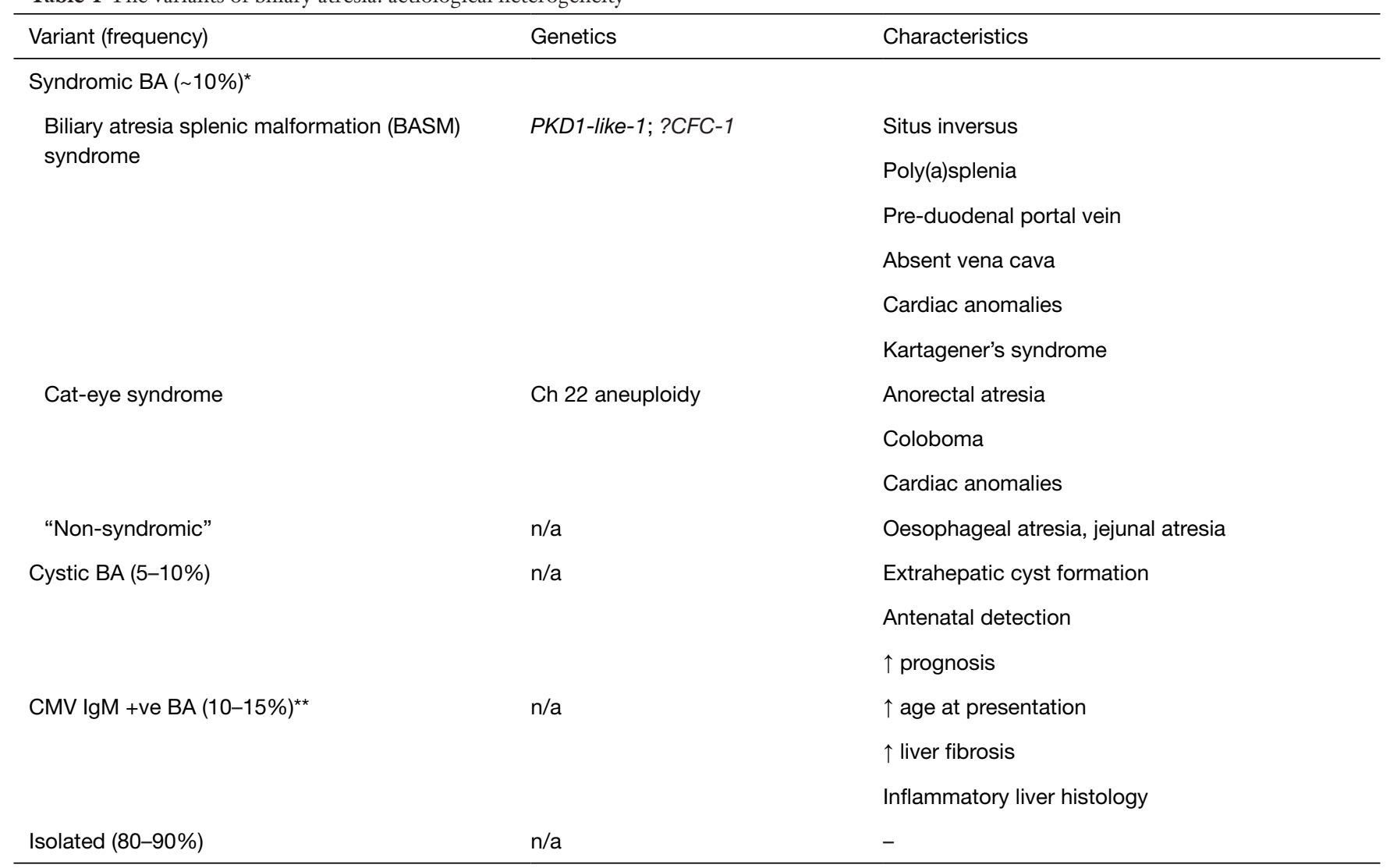

*, prevalence much lower in Asian series. ${ }^{\star *}$, prevalence much higher in Asian series. BA, biliary atresia.

to a mobilized jejunal Roux loop. In a small number of usually late-presenting infants ( $>100$ days of age) where this approach may be deemed futile, primary liver transplantation should be strongly considered.

The outcome following KPE is highly variable in the literature and even more so in the real world with effective drainage of bile achieved in around $50 \%$ of children $(15,16)$. Liver transplantation is indicated (obviously if available) for those who do not achieve adequate resolution of jaundice or who are affected by the severity of complications.

The aim of this review is to discuss and focus on adjuvant therapy and treatments designed to improve outcome after KPE.

\section{Adjuvant therapy}

\section{Corticosteroids (Figure 2)}

Inflammation is thought to be a key factor in the pathogenesis of many cases of BA. There is abnormal expression of MHC class 2 antigens, with upregulation of pro-inflammatory cellular adhesion molecules ICAM and VCAM $(17,18)$ in up to $40 \%$ of cases of BA. This is accompanied by a small cell infiltrate of activated CD4 lymphocytes and NK cells (17). Recent work from our laboratory showed that the $\mathrm{T}$ cell infiltrate is predominantly Th1 and Th17 cells with the former having a particular association in those with CMV IgM antibodies (19). This inflammatory response can also be seen with elevated plasma levels of sICAM, sVCAM together with cytokines such as IL-2, IL-4, IL-18, TNF, interferon. This profile is not only present at the time of KPE but for up to 6 months post-operatively and only seems to resolve after cholestasis recovers (20).

Though this explains much of the rationale behind corticosteroid use in BA with the idea being to limit a presumably detrimental inflammatory response. However, it should also be recognised that corticosteroids have a less well-studied choleretic effect which should also be beneficial. 


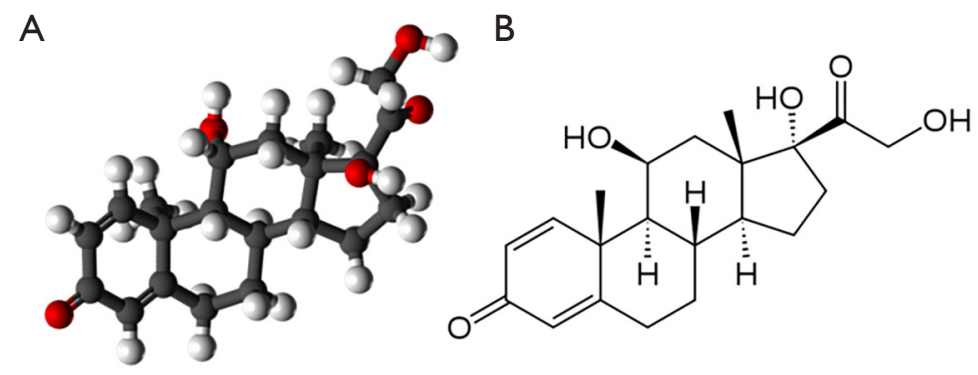

Figure 2 Chemical structure of prednisolone, a synthetic glucocorticoid: (A) ball and stick model (oxygen as red) and (B) steroid ring C21H28O5. [(A) reproduced under Creative Commons License, originator RingO].

\section{Clinical evidence}

Early clinical studies were reported during the 1980's, describing a so-called 'blast-regimen' and popularized by John Lilly from Denver. This was a short course of a high-dose corticosteroid to try and reverse the effect of cholangitis and restore bile flow (21). Following this a multitude of non-randomized small-scale studies were published (22-24) suggesting benefit in a variety of doses and duration.

The first randomized, double blind, placebo-controlled trial was published in 2007 from two UK centres (London and Leeds) and involved 71 infants (25). In retrospect, the regimen used was low-dose and consisted of a starting dose of $2 \mathrm{mg} / \mathrm{kg} /$ day oral prednisolone, reducing over a 4-week period. The primary outcome measure was defined as clearance of jaundice $(\mathrm{CoJ})$ with secondary outcomes being early liver biochemistry and native liver survival. Though there was no difference in overall $\mathrm{CoJ}$ nor need for transplant at six months there were significant differences in bilirubin levels at 1 month post-KPE. A further (now) open-label study was published in 2013 from London (26), restricted to those infants $<70$ days at KPE and adding a further group with a high-dose regimen (starting at $5 \mathrm{mg} / \mathrm{kg} /$ day). This now showed a significant difference between placebo and steroid groups in terms of $\mathrm{CoJ}$ to normal $(<20 \mu \mathrm{mol} / \mathrm{L})$ levels $(52 \%$ vs. $66 \%, \mathrm{P}=0.037)$. Indeed, a gradation of response could be seen between low and high dose regimens. Other biochemical differences were also observed including reduced AST levels and AST-to-platelet ratio index (APRI) at 1 month post-operatively in the high-dose steroid group. Still, it did not show any change in improved native liver survival or reduce the need for transplantation.

The effects of a high-dose prednisolone regimen were also tested in a placebo controlled trial in the North American multicenter $(\mathrm{n}=14)$ STeroids in biliary Atresia Randomised Trial (START) (27). It compared placebo $(n=70)$ against a regimen of IV methylprednisolone ( $4 \mathrm{mg} / \mathrm{kg} /$ day) for 2 weeks tapering down to oral prednisolone $(2 \mathrm{mg} / \mathrm{kg} /$ day $)$ for a further 9 weeks $(n=70)$. The primary endpoint was serum bilirubin $<1.5 \mathrm{mg} / \mathrm{dL}$ at 6 months post KPE. The secondary outcome measure was native liver survival at 6 months. They reported an overall non-significant increase in CoJ at 6 months ( $49 \%$ vs. 59\%) in the steroid group.

Both of the placebo-controlled studies $(26,27)$ identified a negative effect of increasing age on outcome and subset analysis in the START trial confirmed an increased proportion of those to clear their jaundice (71.8\%), but again not to statistical significance. On review of their study design it appears that it was powered to require a difference of $25 \%$ in the primary outcome measure which was certainly wildly optimistic. This estimation was based upon a previously published American retrospective study (22) with very poor outcomes for its control group.

There is also a large non-randomized cohort study from Shanghai, China (28), which compared the outcome of low and high dose steroids in two consecutive periods 2004-2006 and 2007-2009. In total, 380 ( $n=253$ in high dose group) infants underwent KPE. Although there was a significant difference in mean age at KPE (74 vs. 66 days; $\mathrm{P}=0.03$ ) there was a significant difference in the proportion to clear their jaundice (39\% vs. 53\%) in favour of steroids.

Several systematic reviews have been published (29-31). The most recent meta-analysis was published by Chen et al. in 2015 which looked at 259 patients undergoing steroid therapy post-KPE (31). Of the studies meeting the inclusion criteria two were RCTs and five were observational studies, published from 1968 to 2014. They identified from their analysis that there was a significant difference in jaundice clearance in those where moderate 
A

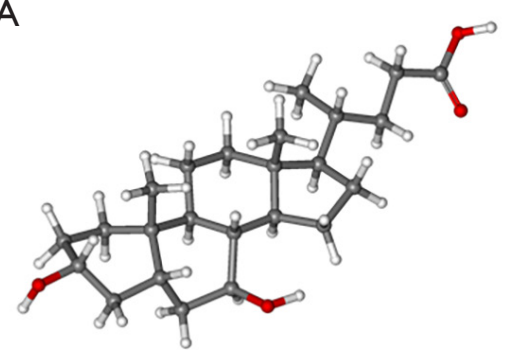

B

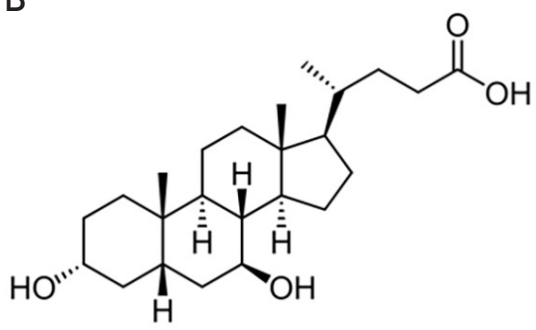

Figure 3 Chemical structure of ursodeoxycholic acid, a secondary bile acid and epimer of chenodeoxycholic acid: (A) ball and stick model (oxygen as red) and (B) C24H40O4. [(A) reproduced under Creative Commons License, originator MarinaVladivostok].

to high-dose steroid versus placebo at 6 months post-KPE. They also suggested that longer regimens failed to elicit any further significant benefit and therefore a shorter course may be more prudent to avoid drug-related complications.

A more recent study from King's College Hospital (32) looked specifically at the "age effect" in a prospective, single-centre, single-surgeon review. One hundred and four infants with BA who underwent KPE at $<70$ days old and received high-dose steroids were included. This group was subdivided into serial age cohorts and $\mathrm{CoJ}$ at 6 months was assessed. This showed a significant trend over time favouring early KPE. Additionally, significant improvement in overall native liver survival occurred in those operated on before 45 days (the median age in the sample). This study for the first time showed that high-dose steroids not only augment jaundice clearance but can also translate to improved native liver survival.

Prednisolone is the most frequently prescribed steroid in most studies $(20,25,27)$ with a usual starting dose of 4 or $5 \mathrm{mg} / \mathrm{kg}$ /day. Some protocols begin this with intravenous methyl prednisolone $(22,27)$ although there is little evidence to suggest this has any extra effect. Dexamethasone has also been recommended by one English centre beginning oral dexamethasone $(0.3 \mathrm{mg} / \mathrm{kg}$ twice daily for 5 days, $0.2 \mathrm{mg} / \mathrm{kg}$ twice daily for 5 days, and $0.1 \mathrm{mg} / \mathrm{kg}$ twice daily for 5 days), beginning on postoperative day 5 (24). The Hannover group in Germany now use rectally-administered budesonide in their current practice (personal communication).

There are many potential side effects of steroids though none has actually been reported in the papers presented so far. Possible side-effects include increased risk of infection, poor wound healing, hyperglycemia, hypertension, gastrointestinal bleeding, poor growth, and an inadequate response to routine immunizations (26). The START trial suggested an increased but non-specific incidence of side effects but were unable to identify anything more tangible (27). Their follow-up study looking at measured growth indices (height, weight and head circumference) did show reduced growth in all groups post KPE but more evident in those with an unsuccessful KPE in the steroid arm (33).

In practice, high-dose steroid use is ubiquitous in the UK, Japan and much of mainland Europe (34). However, its use is probably declining in the USA because of the negative influence of the START trial (27).

\section{Ursodeoxycholic acid (UDCA) (Figure 3)}

UDCA (Ursodiol ${ }^{\mathrm{TM}}$, Actigall ${ }^{\mathrm{TM}}$, URSO $250^{\mathrm{TM}}$ ) is a hydrophilic secondary bile acid that is an established part of treatment for cholestatic conditions in adults $(35,36)$ (Figure 2). Its medicinal benefits were first identified during the Tang Dynasty in China as the traditional drug Shorea. This is derived ultimately from the bile of adult black bears (Figure 4) harvested in somewhat dubious circumstances and contains a high concentration of UDCA.

UDCA has a choleretic effect, increasing hepatic bile flow by up-regulating bile acid transporters, such as Bile Salt Export Protein (BSEP). It is also known to have an immunomodulatory effect and has been shown to decrease cytokine production in primary biliary cholangitis (PBC), alter HLA-class I antigens on hepatocytes $(35,37)$ and suppress immunoglobulin production. There is a documented inverse relationship between increasing serum concentrations of UDCA and decreasing "toxic" endogenous bile salts. This is thought to be protective to hepatocytes and cholangiocytes adding a third rationale for potential benefit in BA. Although there are documented theoretical benefits for UDCA in BA, qualifying evidence is sorely lacking.

There is wealth of evidence supporting its use in PBC and primary sclerosing cholangitis (PSC) with studies 


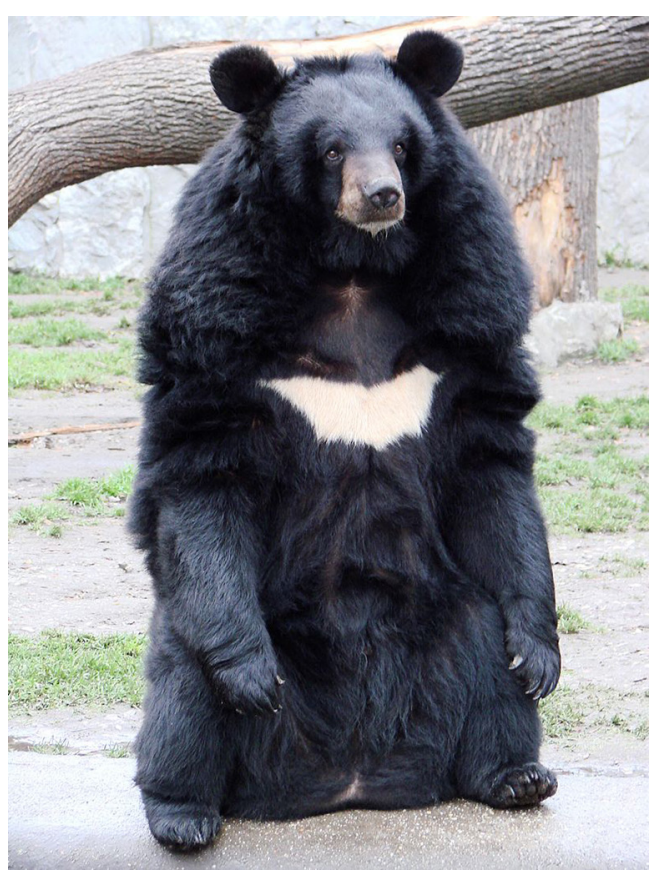

Figure 4 Asian black bear (Ursus thibetanus). Note the distinctive white patch on the chest. (reproduced under Creative Commons License, originator Nicolas Guérin).

reporting improved clinical and biological parameters to reduced need for transplant $(35,36)$. Other conditions suggested to benefit from UDCA are cystic fibrosis and parental nutrition associated cholestasis $(38,39)$.

An early uncontrolled experience from Japan looked at $15 \mathrm{mg} / \mathrm{kg} /$ day UDCA (and additional taurine supplements) in 16 patients post KPE (40). They observed decreases in serum bile acids whether children were still jaundiced or not with restoration to normal values of serum linoleic acid (an essential fatty acid). A prospective uncontrolled crossover trial was performed by a French group (41) to assess the effect of UDCA in stable patients post-KPE. Sixteen children met the inclusion criteria, having cleared their jaundice post-KPE and had been on UDCA $(25 \mathrm{mg} / \mathrm{kg} / \mathrm{day}$ TDS) for at least 1 year. The outcome measures assessed were clinical state (pruritus, ascites, organomegaly, bacterial cholangitis) and biochemical markers (bilirubin, liver enzymes). A discontinuation/reintroduction methodology was used with the hope that as patients were not taking any other medication (such as steroids), any change in outcome measure could be attributed to UDCA cessation/ reintroduction. Thirteen of the 16 children were restarted on UDCA within 6 months of stopping. The main reason for resumption being worsening of liver enzyme profile; with one child having recurrence of jaundice. All of these improved on reintroduction of the drug. Although UDCA showed beneficial effects on liver biochemistry this study did not observe a pronounced effect on clinical status. None of the children experienced increased pruritus, bacterial cholangitis or hepatomegaly on discontinuation of UDCA.

In contrast, there is a larger retrospective study from Egypt (42) which looked at a cohort of 141 infants of which 108 had received UDCA and they suggested that this group had had a worse outcome. However, while not denying the observation this may just reflect their very poor resultsonly $19(13 \%)$ infants overall cleared their jaundice. It seems likely that UDCA was added to try as a last desperate measure to change outcome.

The combination of UDCA and steroids has been subjected to meta-analysis by Qiu et al. who found that there was a significant $(\mathrm{P}=0.0008)$ benefit of the combination in 8 cohort studies though their methodology and inclusion criteria were somewhat opaque (43).

Nevertheless, despite the lack of rigorous evidence particularly the absence of placebo controlled trials the use of UDCA seems universal, certainly it was so in our survey of 19 major European centres (34).

\section{Antibiotics and probiotics}

Cholangitis remains a very serious post-operative complication following KPE, reportedly affecting over 50\% of patients (44). The common causative organisms have been identified as Klebsiella spp., Escherichia coli, Pseudomonas aeruginosa, Escherichia cloacae, A. baumani, Streptococcus mitis and Salmonella typhi (45-48). The mechanism is most likely to be an ascending cholangitis via the Roux loop into the intrahepatic duct system, primitive and distorted though it is. Other contributory mechanisms may also be involved including bacterial overgrowth of bacteria in the gut and a change in the microbiome, translocation from lymphatics and haematogenous spread via the portal vein. The incidence ranges in surgical series between 40-93\% (49,50).

The practice of prescribing prophylactic antibiotics to try and reduce the incidence of cholangitis is extremely variable. There is no consensus as to what drug to give, for what duration and indeed if there is even any clinical benefit. In our survey of European practice (34) the commonest intravenous regimen was a combination of piperacillin-tazobactam (Tazocin ${ }^{\mathrm{TM}}$ ) and gentamicin and the commonest oral prophylactic regimen was based on co-trimoxazole $\left(\right.$ Septrin $\left.^{\mathrm{TM}}\right)$. A recent systematic review by 
Decharun et al. (51) sought to systematically review current evidence on prophylactic antibiotics and their prevention of cholangitis post-KPE. Four studies met the eligibility criteria; one randomized control trial (47) and three retrospective cohort studies (52-54). A lack of high-quality evidence for antibiotic prophylaxis is evident when from 87 abstracts reviewed, only one RCT could be selected. The primary outcome measures were the incidence of cholangitis, recurrent cholangitis and transplant-free survival and a total of 329 patients were included in total (196 receiving antibiotics, 133 control).

There was wide variation in results from the smaller studies; with the largest (54) recruiting from a Dutch national cohort $(\mathrm{n}=214)$. This latter study did not identify any reduction in cholangitis rates, however interestingly it was associated with a higher 4-year transplant free survival rate (54\% in antibiotic group, $34 \%$ in the control group).

The randomized control trial by $\mathrm{Bu}$ et al. (47) concentrated on antibiotic prevention of recurrent cholangitis. Nineteen children, all of whom had had one episode of cholangitis were randomized to receive either trimethoprim-sulfamethoxazole $\left(\right.$ Septrin $\left.{ }^{\mathrm{TM}}\right)(\mathrm{n}=9)$ or oral neomycin $(n=10)$. A historical control group $(n=18)$ of patients who did not receive antibiotics was also used. The authors found that rates of recurrent cholangitis were significantly lower in both antibiotic groups, with no significant difference between the two. Neomycin delayed the first episode of recurrence and was associated with a higher survival rate.

The use of probiotics appears widespread in some countries (e.g., Japan) with the theoretical mechanism being change of the microbiome to one that's less pathogenic. Actual reports of its use however are limited. So, a Taiwanese group (55) randomized two small $(\mathrm{n}=10)$ anicteric groups to 6 months of oral neomycin or oral Lactobacillus Casei Rbamnosus. Given the statistical limitations of comparing such small numbers there were no differences in the prevalence of cholangitis $(20 \%)$ but both appeared superior to a larger historical control group (rate $\sim 80 \%$ ). Stool cultures showed a reduction in E. Coli counts with the rise of Lactobacilli.

This review does reinforce the high incidence of cholangitis in children post-KPE and the expectation that most episodes will occur in the first year post-operatively. A sensible approach to duration of antibiotic therapy may be to give them concomitantly with steroids due to immunosuppression risks or if a longer course is desired to stop at 1 year post-KPE when the cholangitis risk significantly decreases.

Native liver survival decreases markedly if there are repeated episodes of cholangitis especially within the first 2 years of surgery. In one recent study of 76 infants, Koga et al. showed that liver transplantation was ultimately required by all jaundice-free children who had had cholangitis within 3 months of the KPE (56). Our own data from King's College Hospital also supports this interpretation of the important consequences of cholangitis to native liver survival. Thus, in a cohort of 90 infants who achieved $\mathrm{CoJ}$ (66\% of original cohort), 20 (22\%) still underwent LT by 2 years of age. Multivariate analysis showed that cholangitis (any episode but particularly multiple episodes) were significantly related to poor outcome.

Cholangitis occurring after some years of infection-free life in children who have cleared their jaundice should make one suspicious of an actual mechanical problem with the Roux loop (45). These children should be fully investigated with radionuclide imaging, ultrasound and MRCP. Balloon enteroscopy may also have a diagnostic role enabling visualization of the Roux limb. Dilatation proximal to the retrocolic tunnel is the key diagnostic feature and if there is sufficient evidence of this then laparotomy should be embarked upon.

\section{Antiviral therapy (AVT): ganciclovir \& valganciclovir}

Cytomegalovirus IgM +ve associated BA has been proposed as a distinct group of infants with a different aetiology and a worse prognosis $(11,12)$. Perinatal viral exposure may lead to destruction of fully formed bile ducts. This may either be via deleterious effects of the hepatotropic virus itself or a secondary autoimmune reaction $(18,57,58)$.

In a prospective cohort study, Zani et al. (11) clearly identified CMV IgM +ve status as a negative prognostic factor. This single center cohort study identified $20 \mathrm{CMV}$ $\operatorname{IgM}+$ ve infants and compared a range of observations (biochemical, radiological, histological) to a control group of $111 \mathrm{CMV}$-ve infants over a 7 years period. In terms of demographics the CMV IgM +ve patients were older at time of KPE and a more ethnically diverse group with most patients from a non-Caucasian background. They were found have significantly worse preoperative liver function, a higher APRI, more splenomegaly, more inflammation and fibrosis on histology. Postoperatively the CMV IgM +ve infants had significantly reduced rates of jaundice clearance and higher mortality rate. Of note, immunohistochemical 
staining from either liver or resected biliary tissue at $\mathrm{KPE}$ was not able to confirm the presence of the virus in any patient. This difficulty in proving active presence of CMV has been noted in previous studies and Zani et al. hypothesized that it may imply clearance of the virus by the time of KPE. One question which was left unanswered was the time of acquisition of the virus as neither maternal nor neonatal serology were available.

AVT has been suggested for CMV IgM +ve BA (12). It is known to be effective for congenital CMV infection-a much more common entity that the BA variant (59). Thus, ganciclovir was used in 100 infants with congenital CMV disease affecting their central nervous system (59) and shown to reduced disease progression. Its use was however associated with myelosuppression (neutropenia) in about two-thirds. Its use in patients with BA, by contrast, is still poorly reported.

Fischler et al. (60) from Stockholm aimed to describe the effect of IV ganciclovir on patients with CMV-associated cholestasis. They identified six patients in their tertiary referral center with CMV infection (CMV IgM +ve/ CMV in urine). Of these six, two had BA, one had septooptic dysplasia and three had intrahepatic cholestasis attributed to CMV hepatitis. One of the BA patients was diagnosed at $<3$ weeks of age suggesting congenital infection. Ganciclovir was given at $5 \mathrm{mg} / \mathrm{kg}$ BD for 2 weeks then reduced to $5 \mathrm{mg} / \mathrm{kg}$ daily thereafter. The first BA patient received ganciclovir pre-KPE for 3 weeks then post-KPE for a further 2 weeks. The second BA patient had an isolated 4 weeks course. The response was measured biochemically, virologically and by clinical outcome. The results in this very small group of CMV BA patients treated with ganciclovir were however unclear. The first patient displayed improvement in markers of cholestasis. Unfortunately, the second infant did not benefit from KPE and subsequently died from complications following a liver transplant. The authors noted that the concomitant use of UDCA may have influenced results but acknowledge that successful surgery is the biggest factor in improved cholestasis and outcome.

Shah et al. from Mumbai, India report success using oral valganciclovir for CMV-associated BA in one case (61). One patient who had not cleared their jaundice a month post KPE was given a 6 weeks course of valganciclovir. The patient's bilirubin and CMV viral load subsequently improved to normal values.

We have recently published our experience with adjuvant therapy directed at CMV (12). We looked at 36 infants with CMV IgM +ve BA of which 8 had specific AVT (ganciclovir and/or valganciclovir) and compared them to a control group of 28 who did not. CoJ to normal values post-KPE was higher in the AVT group compared to controls $(75 \%$ vs. $21 \%, \mathrm{P}=0.009)$. Native liver survival also improved $(75 \%$ vs. $25 \%$ at 2 years; $\mathrm{P}=0.04)$ but overall survival was similar $(\mathrm{P}=0.24)$. Although our experience is highly suggestive of an effect of AVT it is not conclusive and we await the experience of others. One thing is obvious however is the need to actually measure CMV antibodies and when positive, viral load to try and gauge clinical relevance. Only these could guide empirical AV'T yet rational use.

\section{Vitamin supplementation}

BA is associated with nutritional deficiencies due to reduced intraluminal bile acids. These acids are essential, and must be present at the critical micellar concentration for the adequate absorption of fat and fat-soluble vitamins (A, D, E, K). There are multiple potential consequences of fat-soluble vitamin deficiency such as rickets, fractures and coagulopathy. An essential part of the care for patients with BA is recognizing nutritional deficiencies and providing adequate oral replacement. Venkat et al. (62) suggests that total bilirubin can be used as an effective surrogate for fat-soluble vitamin insufficiency to guide treatment.

A recent paper from King's College Hospital by $\mathrm{Ng}$ et al. (63) focused specifically on vitamin D deficiency in BA. This single-centre retrospective review investigated the pre- and post-KPE levels of 25 hydroxyvitamin D (25 OHVD), liver and bone biochemistry in 129 infants. Each child received high-dose intramuscular vitamin D (30,000 IU or 60,000 in the presence of rickets) followed by oral supplementation post-operatively. Those who were still vitamin $\mathrm{D}$ deficient on monthly blood check received further IM dosing (10,000 IU/kg). Children were followed up at 1, 4, 6 and 12 months. The authors found that vitamin D deficiency was invariable in infants with BA on presentation and that despite supplementation deficiency continued to be a problem post-KPE. This was noted especially in those who were still jaundiced. The authors postulated several theories for this, including poor absorption secondary to on-going cholestasis and lack of intestinal bile acids, and an inadequate metabolism due to hepatocyte dysfunction, possibly exacerbated by ethnicity and even season of birth. 


\section{Intravenous immunoglobulin (IVIG) therapy}

The inflammatory component of BA has already been highlighted and is a clear target for adjuvant therapy. Whereas steroid use has been studies for many years and arrived at placebo-controlled trials, the use of immunoglobulins has not. Intravenous immunoglobulin therapy is known to reduce inflammatory cytokines and increase anti-inflammatory regulatory $\mathrm{T}$ cells (64) and is an established part of treatment in multiple autoimmune, immunodeficiency and inflammatory conditions (65). Fenner et al. (66) using a rhesus-rotavirus model of BA in mice showed that there were diminished bilirubin levels together with less expression of VCAM-1 on portal epithelium and cytokine production by $\mathrm{T}$ cells using highdose IgG therapy.

The Post-Portoenterostomy in Infants with Biliary Atresia (PRIME) (https://clinicaltrials.gov/ct2/show/ NCT01854827) trial looked primarily at safety elements of IVIG (67). This was an open-label multicentre phase $1 \& 2$ trial assessing the feasibility, tolerability and safety of intravenous IG post-KPE. Three doses of $(1 \mathrm{~g} / \mathrm{kg})$ of IVIG $\left(\right.$ Gamunex $\left.^{\circledR}\right)$ were given over a 60 -day period postKPE. Twenty-nine infants were recruited and compared to 64 recruited in the same group's START placebo trial. The results showed no difference in post-operative $\mathrm{CoJ}(35 \%$ $v s .45 \%$ at 1 year) nor need for transplantation, indeed if anything it appeared to be increased in the IVIG group.

A retrospective experience of the use of IVIG was reported from Wuhan, China in a different setting-that of intractable cholangitis post-KPE (68). Patients $(n=16)$ were given IVIG $(400 \mathrm{mg} / \mathrm{kg} /$ day) as an add-on therapy after 3 days conventional antibiotics (meropenem, metronidazole) and steroids (prednisone) if there was no response and controlled with cases who did not have IVIG $(n=13)$. Actual evidence of benefit was difficult to determine as the reasons for therapy were not clear but there was claimed reduction in duration of fever, shorter hospital stay and perhaps increased duration until the next episode. Notably in both groups the ultimate death rate was $>50 \%$.

\section{Chinese herbs and Kampo medicine}

BA is characterised by relatively early-onset aggressive hepatic fibrosis which leads ultimately to cirrhosis. The early response appears to one epithelial-mesenchymal transition (EMT) of biliary epithelial cells into myofibroblasts and may be regulated by TGF- $\beta$, characterised by transition of cadherins, expression of vimentin and $\alpha$-SMA and perhaps under the influence of various microRNAs (e.g., miR-29a and miR-29b1) (69,70). This may lead later to clinically significant portal hypertension, the development of varices, and less commonly ascites. Modulation or even abbreviation of this pathological process would have immense benefit but so far, any actual evidence of effect of any agent seems elusive.

Both Japanese and Chinese centres routinely prescribe the Chinese herb mixture "Inchinko-to" to infants post-KPE and one of the claimed benefits includes inhibition of apoptosis and inhibition of liver fibrosis (70,71). For instance, Tamura et al. (72) reported a prospective study of 21 children postKPE who had cleared their jaundice but who had persistent elevated liver enzymes and GGT. Inchinko-to was given to 12 for up to 3 years while the remainder persisted in their standard regimen without any herb. Liver enzymes, bile acids and markers of liver fibrosis were measured sequentially. There were no side effects of treatment and in the Inchinkoto group, markers of liver fibrosis (e.g., hyaluronic acid) were significantly decreased at 1 and 3 years without change in liver enzymes, bile acids or bilirubin.

\section{Obeticholic acid}

Obeticholic acid (OCA) is a farnesoid $\mathrm{X}$ receptor (FXR) agonist and a modified bile acid that is derived from the primary bile acid chenodeoxycholic acid (CDCA), the natural human FXR ligand. FXR being a nuclear receptor expressed at high levels in the liver (hepatocytes and Kupffer cells) and ileum. Activation in the ileum releases FGF-19 into portal blood which in turn down-regulates endogenous bile acid production in the liver, increases BESP and reduces the bile acid pool while increasing their conjugation. Activation of FXR also inhibits the nuclear factor $\kappa \mathrm{B}(\mathrm{NF}-\kappa \mathrm{B})$-mediated induction of inflammatory mediators, together with TNF- $\alpha$, IL-17, and IFN- $\gamma$ in lymphocyte-enriched human lamina propria cells (73) and may have an anti-fibrotic action (73).

Its principle use has been in the adult conditions of $\mathrm{PBC}$, PSC and non-alcoholic steatohepatitis (NASH) with some significant improvements in liver biochemistry particularly in ALP but less so for bilirubin $(74,75)$. OCA is currently being evaluated for use in BA in a Phase II open-label dosefinding format.

\section{Miscellaneous \& novel approaches}

Phenobarbitone and its derivatives have a long history 
of empirical use in paediatric hepatology and have been within our King's College Hospital protocol since the 1970s (prescribed as phenobarbitone $15 \mathrm{mg}$ daily increasing to $45 \mathrm{mg}$ in steps of $15 \mathrm{mg}$ /week). Similarly, the bile acid sequestrant, cholestyramine (1 sachet t.d.s.) as also been a part of our protocols, again empirically without any real evidence of benefit. Its use probably started following publication of a randomized French trial of 80 infants post$\mathrm{KPE}$ looking at both agents individually together with a comparative control group (76). Ironically, no significant differences were identified which was obviously lost in translation!

The entero-hepatic recirculation of bile acids is a key physiological mechanism for a stable bile acid pool. However, chronic cholestasis causes reduction in luminal conjugated bile acids and up-regulation of ileal bile acid transporters (IBAT) leading to marked increase in the serum levels of bile acids. In the genetic conditions of Alagille's syndrome and Progressive Familial intrahepatic Cholestasis there is marked cholestasis typically from the neonatal period which in some cases responds to surgical bile acid depletion. Recently there have been trials of IBAT inhibitors (e.g., maralixibat) in Alagille's syndrome to try and achieve the same effect, though with indeterminate results (77).

$\mathrm{N}$-acetylcysteine (NAC), currently in widespread use in scenarios of paracetamol (acetaminophen) overdose is currently subject to a phase 2 trial in the immediate postKPE period (78). This will be administered intravenously for the first 7 days at $150 \mathrm{mg} / \mathrm{kg} /$ day. NAC is a precursor to glutathione which has been shown to be depleted in some animal models of BA but is also remarkably choleretic. Its primary outcome measure unusually is normalization of total serum bile acids within 24 weeks, rather than bilirubin levels.

\section{Conclusions}

There is a huge unmet need for novel adjuvant therapy for BA (79). The potential for better results from KPE alone has almost certainly reached its limits in centres with experienced surgeons and a high throughput of cases. A better understanding of aetiological heterogeneity should allow targeted therapy in some (e.g., AVT in CMV IgM $+v e$ infants) and a more nuanced appreciation of the role of inflammation may allow more selected application of steroids. The first year after KPE is also a crucial period in those who have cleared their jaundice because they have so much potential to go on to adult life in possession of their own livers. A better understanding of the role of the microbiome, susceptibility to cholangitis and the advent of truly damaging liver fibrosis probably should be the targets for the next decade.

\section{Acknowledgments}

We acknowledge the major clinical contribution of the paediatric hepatologists at King's College Hospital, London to the care of these infants described in this review and specifically Prof. N Hadzic, Prof. Alastair Baker, Prof. Anil Dhawan and Dr. Marianne Samyn.

Funding: None.

\section{Footnote}

Conflicts of Interest: Both authors have completed the ICMJE uniform disclosure form (available at http://dx.doi. org/10.21037/tp.2016.10.08). MD has acted as consultant for Intercept pharmaceuticals (obeticholic acid) and Mirum pharmaceuticals (maralixibat). JB has no conflicts of interest to declare.

Ethical Statement: The authors are accountable for all aspects of the work in ensuring that questions related to the accuracy or integrity of any part of the work are appropriately investigated and resolved.

Open Access Statement: This is an Open Access article distributed in accordance with the Creative Commons Attribution-NonCommercial-NoDerivs 4.0 International License (CC BY-NC-ND 4.0), which permits the noncommercial replication and distribution of the article with the strict proviso that no changes or edits are made and the original work is properly cited (including links to both the formal publication through the relevant DOI and the license). See: https://creativecommons.org/licenses/by-nc-nd/4.0/.

\section{References}

1. Davenport M. Biliary atresia. Semin Pediatr Surg 2005; 14:42-8.

2. Lin YC, Chang MH, Liao SF, et al. Decreasing rate of biliary atresia in Taiwan: a survey, 2004-2009. Pediatrics 2011;128:e530-6.

3. Wada H, Muraji T, Yokoi A, et al. Insignificant seasonal and geographical variation in incidence of biliary atresia 
in Japan: a regional survey of over 20 years. J Pediatr Surg 2007;42:2090-2.

4. Livesey E, Cortina Borja M, Sharif K, et al. Epidemiology of biliary atresia in England and Wales (1999-2006). Arch Dis Child Fetal Neonatal Ed 2009;94:F451-5.

5. Chardot C, Carton M, Spire-Bendelac N, et al. Epidemiology of biliary atresia in France: a national study 1986-96. J Hepatol 1999;31:1006-13.

6. Jimenez-Rivera C, Jolin-Dahel K, Khiera S, et al. International incidence and outcomes of biliary atresia. J Pediatr Gastroenterol Nutr 2013;56:344-54.

7. Davenport M. Biliary atresia: From Australia to the zebrafish. J Pediatr Surg 2016;51:200-5.

8. Davenport M, Savage M, Mowat AP, et al. Biliary atresia splenic malformation syndrome: an etiologic and prognostic subgroup. Surgery 1993;113:662-8.

9. Davenport M, Tizzard SA, Underhill J, et al. The biliary atresia splenic malformation syndrome: a 28 year single center retrospective study. J Pediatr 2006;149:393-400.

10. Caponcelli E, Kinsely AS, Davenport M. Cystic biliary atresia: an etiologic and prognostic subgroup. J Pediatr Surg 2008;43:1619-24.

11. Zani A, Quaglia A, Hadzic N, et al. Cytomegalovirus associated biliary atresia: an aetiological and prognostic subgroup. J Pediatr Surg 2015;50:1739-45.

12. Parolini F, Hadzic N, Davenport M. Adjuvant therapy of cytomegalovirus IgM + ve associated biliary atresia: Prima facie evidence of effect. J Pediatr Surg 2019;54:1941-5.

13. Harpavat S, Finegold MF, Karpen SJ. Patients with biliary atresia have elevated direct/conjugated bilirubin levels shortly after birth. Pediatrics 2011;128:e1428-33.

14. Lee JY, Sullivan K, El Demellawy D, et al. The value of preoperative liver biopsy in the diagnosis of extrahepatic biliary atresia: A systematic review and meta-analysis. J Pediatr Surg 2016;51:753-61.

15. Davenport $M$, Ong E, Sharif K, et al. Biliary atresia in England and Wales: results of centralization and new benchmark. J Pediatr Surg 2011;46:1689-94.

16. Superina R, Magee JC, Brandt ML, et al. The anatomic pattern of biliary atresia identified at time of Kasai hepatoportoenterostomy and early postoperative clearance of jaundice are significant predictors of transplant-free survival. Ann Surg 2011;254:577-85.

17. Davenport M, Gonde C, Redkar R, et al. Immunohistochemistry of the liver and biliary tree in extrahepatic biliary atresia. J Pediatr Surg 2001;36:1017-25.

18. Barnes BH, Tucker RM, Wehrmann F, et al. Cholangiocytes as immune modulators in rotavirus- induced murine biliary atresia. Liver Int 2009;29:1253-61.

19. Hill R, Quaglia A, Hussain M, et al. Th-17 cells infiltrate the liver in human biliary atresia and are related to surgical outcome. J Pediatr Surg 2015;50:1297-303.

20. Narayanaswamy B, Gonde C, Tredger JM, et al. Serial circulating markers of inflammation in biliary atresia -evolution of the post operative inflammatory process. Hepatology 2007;46:180-7.

21. Karrer FM, Lilly JR. Corticosteroid therapy in biliary atresia. J Pediatr Surg 1985;20:693-5.

22. Meyers RL, Book LS, O'Gorman MA, et al. High-dose steroids, ursodeoxycholic acid and chronic intravenous antibiotics improve bile flow after Kasai procedure in infants with biliary atresia. J Pediatr Surg 2003;38:406-11.

23. Muraji T, Higashimoto Y. The improved outlook for biliary atresia with corticosteroid therapy. J Pediatr Surg 1997;32:1103-6.

24. Stringer MD, Davison SM, Rajwal SR, et al. Kasai portoenterostomy - 12 year experience with a novel adjuvant therapy regimen. J Pediatr Surg 2007;42:1324-8.

25. Davenport $M$, Stringer MD, Tizzard SA, et al. Randomised, double blind, placebo-controlled trial of corticosteroids after Kasai portoenterostomy for biliary atresia. Hepatology 2007;46:1821-7.

26. Davenport M, Parsons C, Tizzard S, et al. Steroids in biliary atresia: single surgeon, single centre, prospective study. J Hepatol 2013;59:1054-8.

27. Bezerra JA, Spino C, Magee JC, et al. Use of corticosteroids after hepatoportoenterostomy for bile drainage in infants with biliary atresia: the START randomised control trial. JAMA 2014;311:1750-9.

28. Dong R, Song Z, Chen G, et al. Improved outcome of biliary atresia with postoperative high-dose steroid. Gastroenterol Res Pract 2013;2013:902431.

29. Sarkhy A, Schreiber RA, Milner RA, et al. Does adjuvant steroid therapy post-Kasai portoenterostomy improve outcome of biliary atresia? Systematic review and metaanalysis. Can J Gastroenterol 2011;25:440-4.

30. Zhang D, Yang HY, Zhao G, et al. Postoperative steroids after Kasai Portoenterostomy for biliary atresia: a metaanalysis. Int J Surg 2014;12:1203-9.

31. Chen Y, Nah S, Chiang L, et al. Postoperative steroid therapy for biliary atresia: systematic review and metaanalysis. J Pediatr Surg 2015;50:1590-94.

32. Tyraskis A, Davenport M. Steroids after Kasai procedure for biliary atresia: the effect of age at Kasai portoenterostomy. Pediatr Surg Int 2016;32:193-200.

33. Alonso EM, Ye W, Hawthorne K, et al. Impact of steroid 
therapy on early growth in infants with biliary atresia: the multicenter Steroids in Biliary Atresia Randomized Trial. J Pediatr 2018;202:179-185.e4.

34. Wong ZH, Davenport M. What happens after Kasai for biliary atresia? a European multicenter survey. Eur J Pediatr Surg 2019;29:1-6.

35. Poupon RE, Balkau B, Eschwege E, et al. A multicentre controlled trial of ursodiol for the treatment of primary biliary cirrhosis. The UDCA-PBC Study Group. N Engl J Med 1994;330:1342-7.

36. Mitchell SA, Bansi DS, Hunt N, et al. A preliminary trial of high dose ursodeoxycholic acid as a therapy for patients with primary sclerosing cholangitis. Gastroenterology 2001;121:900-7.

37. Beuers U, Boyer JL, Paumgartner G. Ursodeoxycholic acid in cholestasis: potential mechanisms of action and therapeutic applications. Hepatology 1998;28:1449-53.

38. Colombo C, Battezzati PM, Podda M, et al. Ursodeoxycholic acid for liver disease associated with cystic fibrosis: a double blind multicentre trial: the Italian group for the study of ursodeoxycholic acid in cystic fibrosis. Hepatology 1996;23:1484-90.

39. De Marco G, Sordino D, Bruzzese E, et al. Early treatment with ursodeoxycholic acid for cholestasis in children on parenteral nutrition because of primary intestinal failure. Aliment Pharmacol Ther 2006;24:387-94.

40. Yamashiro Y, Ohtsuka Y, Shimizu T, et al. Effects of ursodeoxycholic acid treatment on essential fatty acid deficiency in patients with biliary atresia. J Pediatr Surg 1994;29:425-8.

41. Willot S, Uhlen S, Michaud L, et al. Effect of ursodeoxycholic acid on liver function in children after successful surgery for biliary atresia. Pediatrics 2008;122:e1236-41.

42. Kotb MA. Review of historical cohort: ursodeoxycholic acid in extrahepatic biliary atresia. J Pediatr Surg 2008;43:1321-7.

43. Qiu JL, Shao MY, Xie WF, et al. Effect of combined ursodeoxycholic acid and glucocorticoid on the outcome of Kasai procedure: A systematic review and meta-analysis. Medicine (Baltimore) 2018;97:e12005.

44. Rothenberg SS, Scroter GP, Karrer FM, et al. Cholangitis after the Kasai operation for biliary atresia. J Pediatr Surg 1989;24:729-32.

45. Houben C, Phelan S, Davenport M. Late-presenting cholangitis and Roux loop obstruction after Kasai portoenterostomy for biliary atresia. J Pediatr Surg 2006;41:1159-64.
46. Wong KK, Fan AH, Lan LC, et al. Effective antibiotic regime for postoperative acute cholangitis in biliary atresia--an evolving scene. J Pediatr Surg 2004;39:1800-2.

47. Bu LN, Chen HL, Chang CJ, et al. Prophylactic oral antibiotics in prevention of recurrent cholangitis after the Kasai portoenterostomy. J Pediatr Surg 2003;38:590-3.

48. Ernest van Heurn LW, Saing H, Tam PK. Cholangitis after hepatic portoenterostomy for biliary atresia: a multivariate analysis of risk factors. J Pediatr 2003;142:566-71.

49. Luo Y, Zheng S. Current concept about postoperative cholangitis in biliary atresia. World J Pediatr 2008;4:14-9.

50. Lee JY, Lim LT, Quak SH, et al. Cholangitis in children with biliary atresia: health-care resource utilisation. J Paediatr Child Health 2014;50:196-201.

51. Decharun K, Leys CM, West KW, et al. Prophylactic antibiotics for the prevention of cholangitis in patients with biliary atresia status post Kasai portoenterostomy: a systematic review. Clin Pediatr (Phila) 2016;55:66-72.

52. Lally KP, Kanegaye J, Matsumura M, et al. Perioperative factors affecting the outcome following repair of biliary atresia. Pediatrics 1989;83:723-6.

53. Wu ET, Chen HL, Ni YH, et al. Bacterial cholangitis in patients with biliary atresia: impact on short-term outcome. Pediatr Surg Int 2001;17:390-5.

54. de Vries W, de Langen ZJ, Groen H, et al. Biliary atresia in the Netherlands: outcome of patients diagnosed between 1987 and 2008. J Pediatr 2012;160:638-44.e2.

55. Lien TH, Bu LN, Wu JF, et al. Use of Lactobacillus Casei Rhamnosus to prevent cholangitis in biliary atresia after Kasai Operation J Pediatr Gastroenterol Nutr 2015;60:654-8

56. Koga H, Wada M, Nakamura H, et al. Factors influencing jaundice-free survival with the native liver in postportoenterostomy biliary atresia patients: results from a single institution. J Pediatr Surg 2013;48:2368-72.

57. Petersen C, Davenport M. Aetiology of biliary atresia: what is actually known? Orphanet J Rare Dis 2013;8:128.

58. Mack CL. The pathogenesis of biliary atresia: evidence for a virus-induced autoimmune disease. Semin Liver Dis 2007;27:233-42.

59. Kimberlin DW, Lin CY, Sánchez PJ, et al. Effect of ganciclovir therapy on hearing in symptomatic congenital cytomegalovirus disease involving the central nervous system: a randomized, controlled trial. J Pediatr 2003;143:16-25.

60. Fischler B, Casswall TH, Malmborg P, et al. Ganciclovir treatment in infants with cytomegalovirus infection and cholestasis. J Pediatr Gastroenterol Nutr 2002;34:154-7. 
61. Shah I, Bhatnagar S. Biliary atresia and cytomegalovirus and response to valganciclovir. Indian Pediatr 2012;49:484-6.

62. Venkat VL, Shneider BL, Magee JC, et al. Total serum bilirubin predicts fat-soluble vitamin deficiency better than serum bile acids in infants with biliary atresia. J Pediatr Gastroenterol Nutr 2014;59:702-7.

63. Ng J, Paul A, Wright N, et al. Vitamin D levels in infants with biliary atresia: pre and post Kasai portoenterostomy. J Pediatr Gastroenterol Nutr 2016;62:746-50.

64. Tucker RM, Feldman AG, Fenner EK, et al. Regulatory T cells inhibit Th1 cell-mediated bile duct injury in murine biliary atresia. J Hepatol 2013;59:790-6.

65. Gelfand EW. Intravenous immune globulin in autoimmune and inflammatory diseases. $\mathrm{N}$ Engl J Med 2012;367:2015-25.

66. Fenner EK, Boguniewicz J, Tucker RM, et al. High-dose IgG therapy mitigates bile duct-targeted inflammation and obstruction in a mouse model of biliary atresia. Pediatr Res 2014;76:72-80.

67. Mack CL, Spino C, Alonso EM, et al. A Phase I/ IIa Trial of intravenous immunoglobulin following portoenterostomy in biliary atresia. J Pediatr Gastroenterol Nutr 2019;68:495-501.

68. Dandan Li, Wang P, He Y, et al. Intravenous immunoglobulin for the treatment of intractable cholangitis after Kasai portoenterostomy in biliary atresia patients. Pediatr Surg Int 2018;34:399-404.

69. Xiao Y, Zhou Y, Chen Y, et al. The expression of epithelial-mesenchymal transition-related proteins in biliary epithelial cells is associated with liver fibrosis in biliary atresia. Pediatr Res 2015;77:310-5.

70. Deng YH, Pu CL, Li YC, et al. Analysis of biliary epithelial-mesenchymal transition in portal tract fibrogenesis in biliary atresia. Dig Dis Sci 2011;56:731-40.
71. Iinuma Y, Kubota M, Yagi M, et al. Effects of the herbal medicine inchinko-to on liver function in postoperative patients with biliary atresia - a pilot study. J Pediatr Surg 2003;38:1607-11.

72. Tamura T, Kobayashi H, Yamataka A, et al. Inchin-koto prevents medium-term liver fibrosis In postoperative biliary atresia patients. Pediatr Surg Int 2007;23:343-7.

73. Gadaleta RM, van Erpecum KJ, Oldenburg B, et al. Farnesoid $\mathrm{X}$ receptor activation inhibits inflammation and preserves the intestinal barrier in inflammatory bowel disease. Gut 2011;60:463-72.

74. Carbone M, Harms MH, Lammers WJ, et al. Clinical application of the GLOBE and United Kingdom-primary biliary cholangitis risk scores in a trial cohort of patients with primary biliary cholangitis. Hepatol Commun 2018;2:683-92.

75. Kowdley KV, Vuppalanchi R, Levy C, et al. A randomized, placebo-controlled, phase II study of obeticholic acid for primary sclerosing cholangitis. J Hepatol 2020. doi: 10.1016/j.jhep.2020.02.033.

76. Vajro P, Couturier M, Lemonnier F, et al. Effects of postoperative cholestyramine and phenobarbital administration on bile flow restoration in infants with extrahepatic biliary atresia. J Pediatr Surg 1986;21:362-5.

77. Shneider BL, Spino C, Kamath BM, et al. Placebocontrolled randomized trial of an intestinal bile salt transport inhibitor for pruritus in Alagille syndrome. Hepatol Commun 2018;2:1184-98.

78. Tessier MEM, Shneider BL, Brandt ML, et al. A phase 2 trial of N-Acetylcysteine in biliary atresia after Kasai portoenterostomy. Contemp Clin Trials Commun 2019; 15:100370.

79. Bezerra JA, Wells RG, Mack CL, et al. Biliary atresia: clinical and research challenges for the 21st Century. Hepatology 2018;68:1163-73.
Cite this article as: Burns J, Davenport M. Adjuvant treatments for biliary atresia. Transl Pediatr 2020;9(3):243-255. doi: $10.21037 /$ tp.2016.10.08 